\title{
Interventional Neurology
}

\section{Carotid Stenting for Treatment of Symptomatic Carotid Webs: A Single-Center Case Series}

\author{
Waleed Brinjikji Ronit Agid Vitor M. Pereira \\ Joint Department of Medical Imaging, Toronto Western Hospital, University of \\ Toronto, Toronto, ON, Canada
}

\section{Keywords}

Stent $\cdot$ Carotid artery disease $\cdot$ Stroke

\begin{abstract}
Background and Purpose: Carotid webs are an increasingly recognized cause of acute ischemic stroke, particularly in younger adults. The optimal medical and surgical strategies for managing these lesions have not been well established. We report a single-center case series of carotid stenting for treatment of symptomatic carotid webs. Materials and Methods: Consecutive patients undergoing stent placement for treatment of symptomatic carotid webs were included. Carotid webs were defined as a thin intraluminal filling defect along the posterior wall of the carotid bulb just beyond the carotid bifurcation on CTA. Data were collected on demographic characteristics, antiplatelet management, clinical presentation, imaging findings, treatment characteristics, complications, and stroke recurrence rates. Descriptive statistics are reported. Results: A total of 4 patients were treated. Their mean age was 44 years (range 30-50). Three patients were female and 1 was male. All patients were symptomatic presenting with ipsilateral transient ischemic attacks or stroke. Patients were placed on dual antiplatelet therapy with ticagrelor and aspirin prior to the procedure. There were no ischemic or hemorrhagic complications. Three patients had postoperative bradycardia, 1 of whom required atropine immediately following stenting. No patients had recurrent ischemic events. Conclusions: Stent placement for treatment of carotid webs can be performed safely. Further studies are needed to confirm our findings.




\section{Introduction}

Carotid webs are an increasingly recognized cause of acute ischemic stroke, particularly in younger adult patients. These lesions are defined as endoluminal shelf-like projections at the origin of the internal carotid artery just beyond its bifurcation [1,2]. Carotid webs have also been described as atypical fibromuscular dysplasia, septal fibromuscular dysplasia, pseudovalvular folds and carotid diaphragms $[1,2]$. Histologically, these lesions are distinct from atherosclerotic plaques as they are characterized by a shelf-like projection of fibrous intimal thickening sometimes with a tiny focal dissection into the fibrotic intima $[1,2]$. One recently published study found that up to $10 \%$ of patients aged 60 years and younger have a carotid web ipsilateral to the territory of the ischemic stroke [2].

While these lesions are increasingly recognized as a cause of acute ischemic stroke, little is known regarding the ideal management strategy of patients with carotid webs. Stroke recurrence rates in this patient population are high, up to $25-35 \%$, even with antiplatelet therapy [2, 3]. Prior small series and case reports have reported variable outcomes with aggressive medical management (i.e., dual antiplatelet therapy), carotid endarterectomy, and even carotid stenting as strategies to reduce stroke recurrence rates in this patient population [1-4]. Herein, we report our single-center experience with carotid stenting for treatment of carotid webs.

\section{Materials and Methods}

\section{Patient Population}

Following institutional review board approval, we retrospectively identified all carotid web patients at our institution who underwent treatment of the lesion with carotid stenting. Inclusion criteria were the following: (1) adult patients, (2) CT angiographically confirmed carotid web using previously described criteria, and (3) patient underwent stenting for treatment of the lesion. Patients with atherosclerotic plaques, shelf-like lesions at sites of endarterectomy grafts and cervical carotid dissections were excluded.

\section{Procedure Techniques}

Prior to the procedure, patients were premedicated for 3-5 days with ticagrelor $(90 \mathrm{mg}$ by mouth, twice daily) and aspirin (81 mg by mouth once daily). In general, a short 8-Fr sheath was placed in the groin. Following this, a 6-Fr 80-cm Cook Shuttle Sheath was placed in the common carotid artery. In 2 cases, a SpiderFx filter was advanced across the lesion over a microguidewire and in 2 cases, no filter was used. Under road map guidance, a single Protégé or Wallstent was placed across the lesion. No balloon angioplasty was performed in any cases.

\section{Data Collection and Outcomes}

The following data were collected for each patient: age, gender, symptoms at presentation/indication for treatment, degree of stenosis related to the web, antiplatelet therapy strategy, stent type, use of filter, and use of balloon angioplasty. All CTA examinations were reviewed for confirmation of the presence of a web by a neuroradiologist. Outcomes studied included perioperative complications, restenosis rates, and stroke recurrence.

\section{Statistical Analysis}

Only descriptive statistics are reported. Means are presented with their standard deviations and proportions are presented with their percentages. All statistical analyses were performed using JMP13.0 (www.jmp. com, Cary, NC, USA). 
Interventional

Neurology

Fig. 1. Patient with recurrent right hemispheric transient ischemic attacks. a Axial CTA image demonstrates a web (arrow) of the right carotid bulb which has the appearance of a septum on axial images. b Coronal reconstructed image of the CTA shows the web as a shelflike projection into the carotid bulb (arrow). c This is also demonstrated on the lateral projection right carotid angiogram (arrow). d Post-stenting angiogram shows resolution of the web with a patent right internal carotid artery.

Fig. 2. a Patient with carotid web presenting with large-vessel occlusion. $\mathbf{b}$ Axial reconstruction of an XperCT image performed during intra-arterial contrast injection shows a web with a septum on axial images. c Coronal reconstruction shows the shelflike projection projecting into the right internal carotid artery. d Post-stenting angiogram shows resolution of the web.

\begin{tabular}{l|l}
\hline Intervent Neurol 2018;7:233-240 \\
\hline DOI: 10.1159/000486537 & $\begin{array}{l}\text { @ 2018 S. Karger AG, Basel } \\
\text { www.karger.com/ine }\end{array}$ \\
\hline
\end{tabular}

Brinjikji et al.: Carotid Stenting for Treatment of Symptomatic Carotid Webs: A SingleCenter Case Series
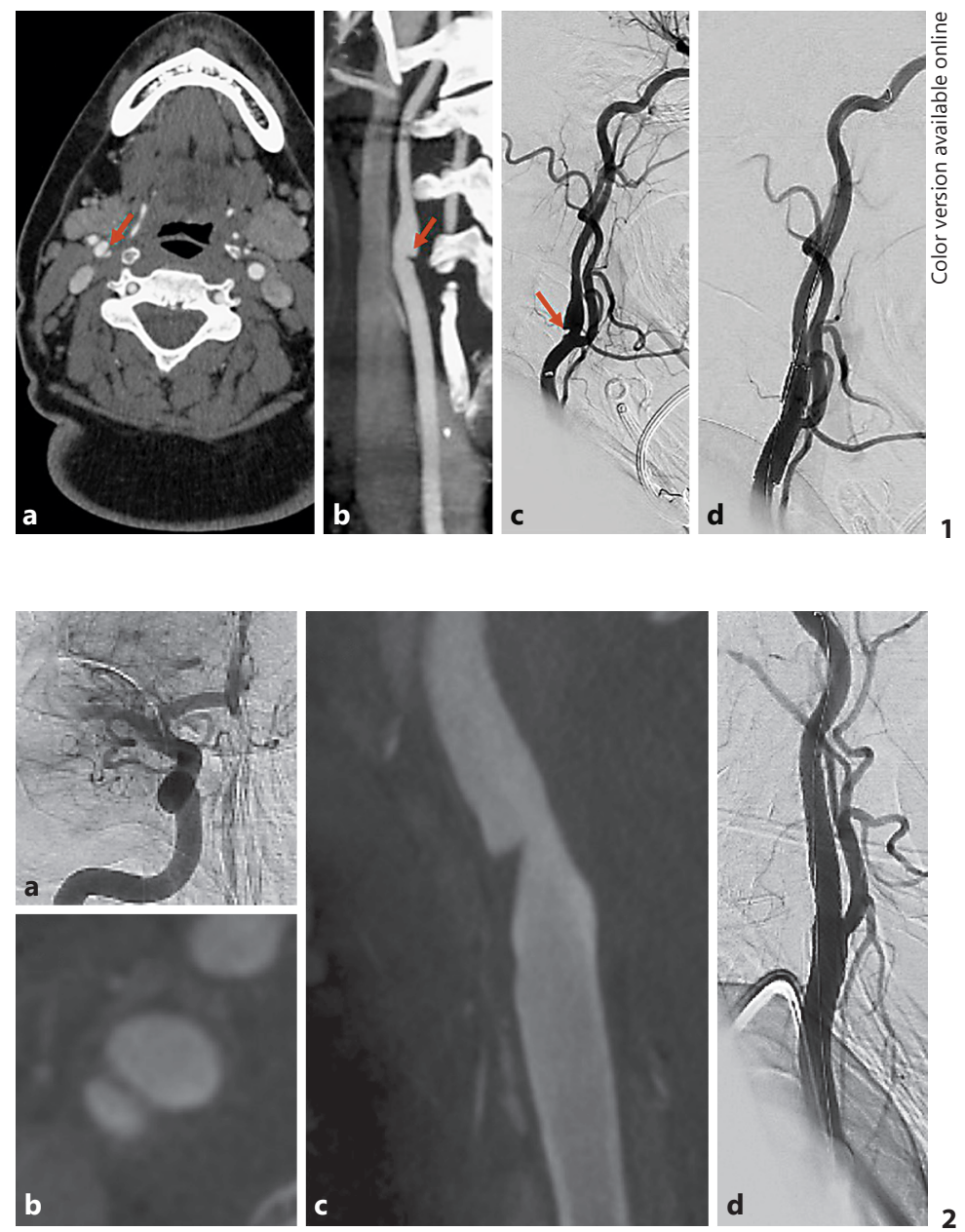

\section{Results}

\section{Patient Characteristics and Presentation}

Four patients were included in this study. Preoperative and intraoperative imaging findings for each patient are shown in Figures 1-4. Mean age was $44 \pm 9.5$ years. Three patients were female (75\%). No patients had atherosclerotic risk factors and no patients had identifiable cardiac causes for stroke. All patients were symptomatic, 3 with recurrent ischemic symptoms: 1 patient presenting with stroke secondary to large-vessel occlusion, 1 patient presenting with recurrent transient ischemic attacks in one territory, and 2 patients presenting with recurrent ischemic strokes in one carotid territory. All patients were treated within 1 week of last presentation.

\section{Imaging}

All patients had CTA evidence of a carotid web. One patient had intraluminal thrombus on CT at the distal aspect of the web. One patient had MRI vessel wall imaging which demonstrated a thin fibrous band and no plaque hemorrhage or enhancement (Fig. 3). On catheter angiography, all 4 patients had stasis of contrast along the shelf of the web. An example of this is shown in Figure 4. 
Interventional
Neurology

Fig. 3. a Patient presenting with an acute left frontal infarct as seen on diffusion-weighted MRI images. CTA (not shown) showed evidence of a carotid web. b Axial MRI T1 FSE black blood vessel wall imaging shows the septum as a thin fibrous band with no associated hemorrhage. c Pretreatment lateral angiogram of the left common carotid artery shows the web as a shelf-like projection with contrast stasis distal to the web. d Post-stenting angiogram shows resolution of the web.

Fig. 4. a Carotid web in a patient presenting with a left insular stroke as demonstrated on noncontrast CT. b Lateral-projection digital subtraction angiography shows the shelf-like web which is also demonstrated on 3D reconstruction (c). d Late-phase carotid angiogram shows stasis of contrast in the shelf distal to the web.

\begin{tabular}{l|l}
\hline Intervent Neurol 2018;7:233-240 \\
\hline DOI: 10.1159/000486537 & $\begin{array}{l}\text { c 2018 S. Karger AG, Basel } \\
\text { www.karger.com/ine }\end{array}$ \\
\hline
\end{tabular}

Brinjikji et al.: Carotid Stenting for Treatment of Symptomatic Carotid Webs: A SingleCenter Case Series
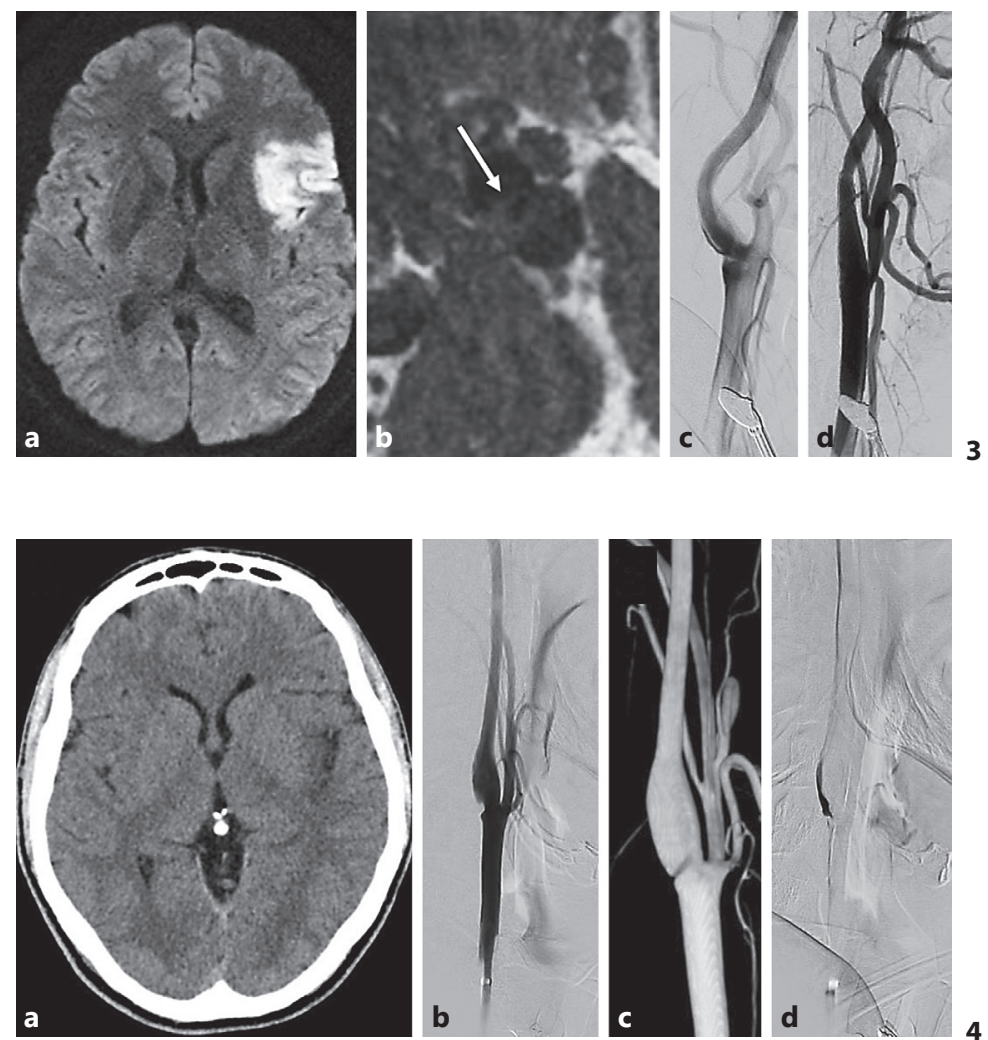

\section{Outcomes}

All patients were successfully treated. In all cases, stenting effectively eliminated the protrusion of the web into the carotid bulb's lumen. No patients suffered ischemic or hemorrhagic complications related to the procedure. Inspection of the filters used in 2 cases demonstrated no debris. The patient with the superimposed thrombus was treated using a distal protection device while on a heparin drip. The thrombus was present at the time of the procedure but inspection of the filter demonstrated no debris and the postoperative angiogram showed no emboli suggesting that the thrombus was apposed between the stent and arterial wall.

During the procedure, 1 patient had mild vasospasm distal to the stent which resolved with $5 \mathrm{mg}$ of intra-arterial verapamil. Three patients experienced post-stenting bradycardia, 1 of whom required short treatment with atropine. Mean clinical and imaging follow-up time was 9 months (range 5-21). There were no cases of restenosis on CTA (performed in all patients) or stroke recurrence.

\section{Discussion}

Our study of 4 symptomatic carotid web patients who underwent carotid stenting demonstrated a number of interesting findings. First, all included patients were relatively young (30-50 years old) with no atherosclerotic risk factors, further enforcing the idea that carotid webs are an important cause of ischemic stroke in younger adults. Second, treatment of these lesions was safe with the only minor complication related to the procedure being transient 
post-stenting bradycardia. No patients had recurrent ischemic events following treatment and no patients had postoperative in-stent stenosis. Larger studies are needed to both confirm our results and determine when treatment of these lesions is best indicated.

Histologically, carotid webs are formed by an abnormal intimal layer of fibrosis and hyperplasia without atherosclerotic plaque [1]. These lesions are similar to those found in patients with fibromuscular dysplasia. On conventional angiography, carotid webs are defined by a shelf or triangular-like projection into the lumen with stagnation and turbulence of blood flow distal to the lesion. The typical CTA appearance of these lesions is a thin intraluminal filling defect along the posterior wall of the carotid bulb just beyond the carotid bifurcation. On axial CTA images, a septum is often seen [1]. Interobserver agreement for identification of webs on CTA is high with kappa values over 0.90 [2]. Examination of these lesions with vessel wall imaging shows focal vessel wall thickening with a thin fibrous band and no enhancement, plaque hemorrhage or other atherosclerosis-type characteristics [5]. One such example of wall imaging is provided in Figure 3.

Carotid webs are an underrecognized cause of ischemic stroke, particularly in younger patients. In a series of 33 patients with cryptogenic stroke who underwent neck CTA, Sajedi et al. [6] found that $21.2 \%$ of patients had a carotid web ipsilateral to the stroke territory compared to only $1.6 \%$ of patients in the control group. In another case-control study of cryptogenic stroke patients, including 164 cases matched by age and gender, Coutinho et al. [2] found that the prevalence of carotid webs in the cryptogenic stroke population was $9.4 \%$ compared to $1.0 \%$ in controls. In general, larger series of symptomatic carotid web patients have found that these lesions are predominantly seen in females with a female:male ratio of 3:1-4:1 and are typically found in younger patients, with the mean age for most series ranging from 40 to 50 years. In our series, all patients were 50 years old or younger and $75 \%$ of patients were female.

Ideal management strategies for patients with carotid webs are not yet clear. The mechanism of stroke from these lesions is thought to be related to stasis of blood flow distal to the carotid web resulting in thrombus formation. An angiographic representation of such stasis is shows in Figure 4. Medical therapies that have been studied in smaller case series include anticoagulation and dual antiplatelet therapy. Stroke recurrence rates in this patient population, even in the setting of antiplatelet therapy, range from 30 to $70 \%$. Some have advocated the use of anticoagulation based on the fact that the mechanism of stroke is due to stasisinduced thrombosis, similar to that seen in strokes related to atrial fibrillation. Others have advocated for the use of endarterectomy to resect the web or carotid stenting [3, 7]. In a series of 25 patients with symptomatic carotid webs, Joux et al. [3] performed endarterectomy in 7 cases with no cases of stroke recurrence or complications. Meanwhile, patients who were treated with antiplatelet therapy had a stroke recurrence rate of $30 \%$ at 2 years. In a series of 24 patients with stroke or transient ischemic attack, Haussen et al. [8] found a recurrence rate of $32 \%$, with 16 patients undergoing stent placement across the web with no complications or recurrent ischemic events. Similarly, in a series of 5 patients with carotid webs and recurrent stroke on medical therapy, 4 patients who underwent endarterectomy were stroke free after 3-7 months of follow-up [1].

Four case series and case reports with a total of 21 patients have reported outcomes of carotid web stenting in patients with symptomatic lesions (Table 1) [4, 8-10]. The largest study to date included 16 patients [8]. All cases were technically successful and there were no complications, recurrences or in-stent stenosis during follow-up periods ranging from 6 months to 8.5 years $[4,9,10]$. Treatment of a carotid web is mostly aimed at flattening the web so that it never becomes a hemodynamic nidus for future ischemic events. Carotid stenting with a self-expanding stent is thought to be an effective treatment for these lesions due to the fact that once wall apposition is achieved, the hemodynamic disturbances distal to 
Brinjikji et al.: Carotid Stenting for Treatment of Symptomatic Carotid Webs: A SingleCenter Case Series

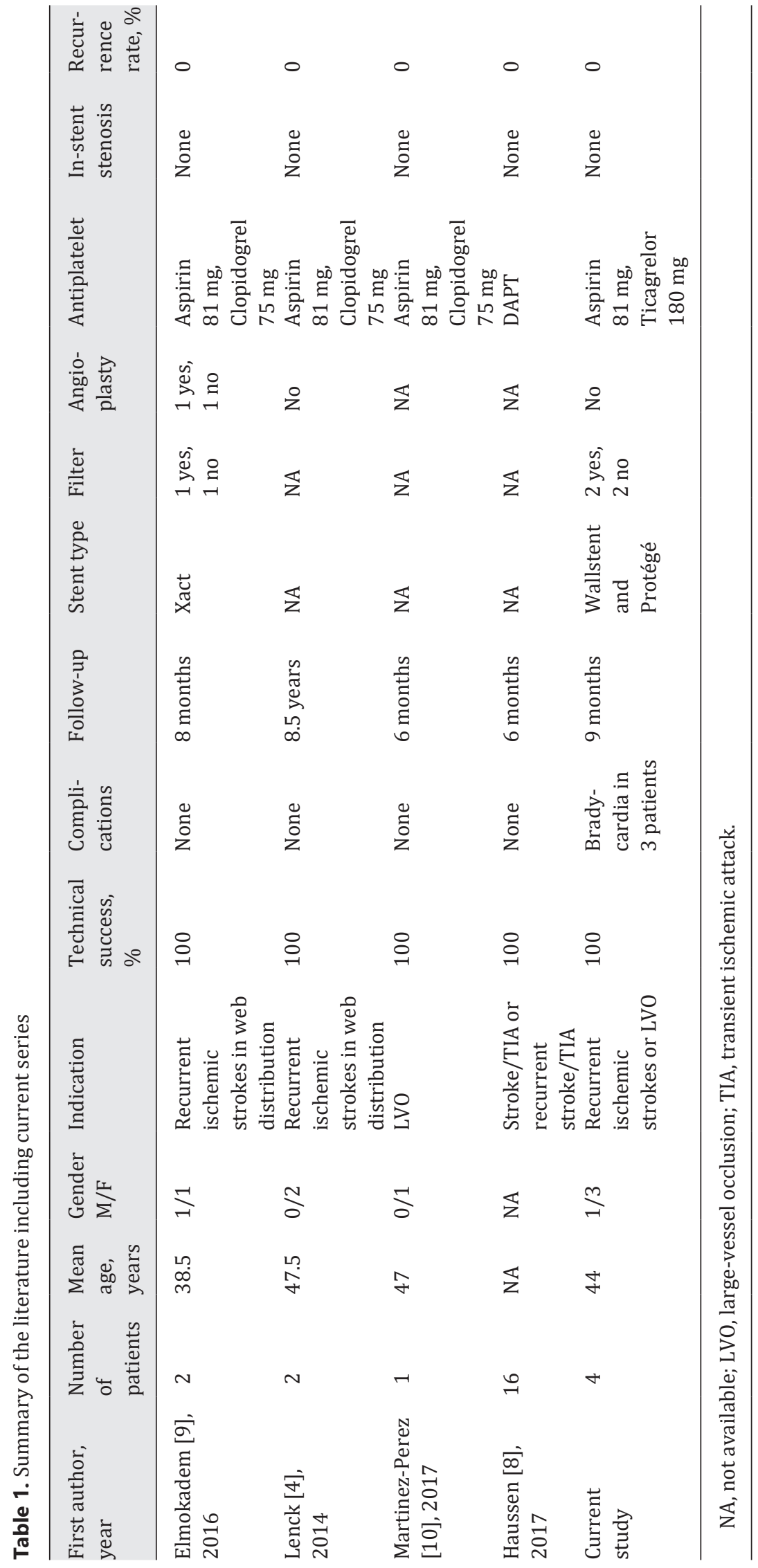


the web are no longer present. In general, balloon angioplasty is not necessary as there is no flow-limiting stenosis. In the absence of CT evidence of intraluminal thrombus, distal protection devices are likely not necessary as there is no plaque disruption. In our series, we found that post-stenting bradycardia and hypotension were relatively common minor complications. In one case, one dose of atropine was required and in no cases did the hypotension and bradycardia last beyond the hospital discharge. The mechanism of hypotension and bradycardia in this patient population is related to the use of a self-expanding stent which is stimulating baroreceptors in a relatively normal carotid artery. It is important for neurointerventionalists to be cognizant of this minor complication.

\section{Limitations}

The main limitation of our study is being a small single-center case series and it is difficult to make any generalizations regarding the safety, efficacy, and utility of carotid stenting in this population. There was no control group on medical therapy so no conclusions can be drawn regarding the relative efficacy of stenting over medical therapy. Another limitation is the lack of long-term follow-up which limits our understanding of the long-term efficacy of this treatment.

\section{Conclusions}

Stent placement for treatment of carotid webs can be performed safely. Further studies are needed to determine if it is an effective tool in reducing stroke recurrence rates in this patient population.

\section{Disclosure Statement}

Waleed Brinjikji is CEO of Marblehead Medical LLC and has patents pending in balloon catheter technologies.

\section{Author Contributions}

W.B., R.A., and V.M.P. made substantial contributions to the conception or design of the work or the acquisition, analysis or interpretation of data for the work; drafting of the work or revising it critically for important intellectual content; and final approval of the version to be published; and are in agreement to be accountable for all aspects of the work in ensuring that questions related to the accuracy or integrity of any part of the work are appropriately investigated and resolved. Data can be made available by contacting the corresponding author via e-mail.

\section{References}

1 Choi PM, Singh D, Trivedi A, Qazi E, George D, Wong J, Demchuk AM, Goyal M, Hill MD, Menon BK: Carotid webs and recurrent ischemic strokes in the era of CT angiography. AJNR Am J Neuroradiol 2015;36:2134-2139.

2 Coutinho JM, Derkatch S, Potvin AR, Tomlinson G, Casaubon LK, Silver FL, Mandell DM: Carotid artery web and ischemic stroke: a case-control study. Neurology 2017;88:65-69.

3 Joux J, Chausson N, Jeannin S, Saint-Vil M, Mejdoubi M, Hennequin JL, Deschamps L, Smadja D, Olindo S: Carotid-bulb atypical fibromuscular dysplasia in young Afro-Caribbean patients with stroke. Stroke 2014;45: 3711-3713.

4 Lenck S, Labeyrie MA, Saint-Maurice JP, Tarlov N, Houdart E: Diaphragms of the carotid and vertebral arteries: an under-diagnosed cause of ischaemic stroke. Eur J Neurol 2014;21:586-593. 
5 Boesen ME, Eswaradass PV, Singh D, Mitha AP, Goyal M, Frayne R, Menon BK: MR imaging of carotid webs. Neuroradiology 2017;59:361-365.

6 Sajedi PI, Gonzalez JN, Cronin CA, Kouo T, Steven A, Zhuo J, Thompson O, Castellani R, Kittner SJ, Gandhi D, Raghavan P: Carotid bulb webs as a cause of "cryptogenic" ischemic stroke. AJNR Am J Neuroradiol 2017;38: 1399-1404.

7 Phair J, Trestman EB, Yean C, Lipsitz EC: Endarterectomy for a symptomatic carotid web. Vascular 2017; 25: 553-556.

8 Haussen DC, Grossberg JA, Bouslama M, Pradilla G, Belagaje S, Bianchi N, Allen JW, Frankel M, Nogueira RG: Carotid web (intimal fibromuscular dysplasia) has high stroke recurrence risk and is amenable to stenting. Stroke 2017;48:3134-3137.

9 Elmokadem AH, Ansari SA, Sangha R, Prabhakaran S, Shaibani A, Hurley MC: Neurointerventional management of carotid webs associated with recurrent and acute cerebral ischemic syndromes. Interv Neuroradiol 2016; 22:432-437.

10 Martinez-Perez R, Lownie SP, Pandey SK, Boulton MR: Stent placement for carotid web. World Neurosurg 2017;98:879.e9-879.e11. 